\title{
What does a First Order Model tell us about PEEP Wave Maneuvers?
}

\author{
B. Laufer ${ }^{1}$, J. Kretschmer ${ }^{1}$, P. D. Docherty ${ }^{2}$, Y. S. Chiew ${ }^{3}$, K. Möller ${ }^{1}$ \\ ${ }^{1}$ Institute of Technical Medicine, Furtwangen University, Villingen-Schwenningen, Germany \\ ${ }^{2}$ Department of Mechanical Engineering, University of Canterbury, Christchurch, New Zealand \\ ${ }^{3}$ School of Engineering, Monash University Malaysia, Bandar Sunway, Malaysia \\ Email: lab@hs-furtwangen.de
}

How to cite this paper: Laufer, B., Kretschmer, J., Docherty, P.D., Chiew, Y.S and Möller, K. (2017) What does a First Order Model tell us about PEEP Wave Maneuvers?. J. Biomedical Science and Engineering, 10, 66-75. https://doi.org/10.4236/jbise.2017.105B008

Received: April 13, 2017

Accepted: May 3, 2017

Published: May 10, 2017

\begin{abstract}
Patients with acute respiratory distress syndrome (ARDS) are currently treatec with a lung protective ventilation strategy and the application of positive end-expiratory pressure (PEEP), sometimes in combination with recruitmen maneuvers. In this study, the respiratory system elastance and airway resistance of each breath before, during and after a specific recruitment maneuver (PEEF wave maneuver) were analyzed in two patient groups, ARDS and control group. A reduction of elastance after the maneuver was observed in ARDS patients. In addition, only healthy lungs exhibited a reduction of the elastance during the course of the maneuver, while the lungs of ARDS patients didn't show that reduction of elastance. The capability of PEEP wave maneuvers to improve lung ventilation was shown and the dynamic behavior of the elastance after the maneuver was illustrated. Healthy lungs adapt faster to changes in mechanical ventilation than the lungs of ARDS patients.
\end{abstract}

\section{Keywords}

ARDS, Positive End-Expiratory Pressure, Recruitment Maneuvers, First Order Model, Lung Mechanics

\section{Introduction}

Acute respiratory distress syndrome (ARDS) captures a variety of pulmonary dysfunctions. It was first described in the late 1960s by Ashbaugh et al. [1]. Due to the wide range of etiology and pathogenesis, ARDS [2] is noted for its complexity and heterogeneity. The causes of ARDS can include pneumonia, sepsis, trauma, asthma, chronic obstructive pulmonary disease (COPD), pancreatitis, burns and near drowning while the pathologic syndromes include edema (alveolar and interstitial) and fibroses [3] [4].

Currently, ARDS remains a major clinical problem with ambiguous understanding of the benefits of different treatment approaches. There is a lack of consensus within the clinical community regarding the optimal treatments for the 
various forms of ARDS. However, there is consensus that mechanical ventilation (MV) is an essential therapy for ARDS patients. The current general MV approach is known as lung protective ventilation. The tidal volumes of this lung protective ventilation are lower $(6 \mathrm{ml} / \mathrm{kg})$ [5] and the positive end-expiratory pressure (PEEP) levels are higher than previously, though beneficial [6]. Studies have reported that these settings in combination with recruitment maneuvers decrease the mortality and the recovery-time of patients [7] [8]. However, other studies have claimed that the influence of PEEP [9] or recruitment maneuvers [10, 11] on outcome / final patient mortality is small.

Different recruitment maneuvers can be used to improve the lung ventilation, in the dataset at hand an incremental PEEP Wave (PW) Maneuver [12] [13], a Super Syringe (SuSy) Maneuver [14], a Static Compliance by Automated Single Steps (SCASS) Maneuver [14], a Low Flow Maneuver [12] [14] and a Dynamic Slice Maneuver were performed in random sequence. We limited this study to the PEEP Wave (PW) Maneuver (Figure 1). During an incremental PEEP wave maneuver a set of five or more breaths are induced by MV with incrementally increasing PEEP levels. Usually the maneuver starts at Zero End-Expiratory Pressure (ZEEP) and 5 steps with increments of 2 mbar are undertaken. The PW maneuver can help to find the optimal PEEP level.

Many studies have been done to determine the optimal lung protective ventilation [5] [15], but the selection of the optimal value for the positive end-expi- ratory pressure (PEEP) level is still a challenge in the treatment of patients with ARDS [3] [4] [7]. Physiological modeling of the respiratory system is one way to counteract that problem and to find out the best possible settings for mechanical ventilation [16] [17] [18].

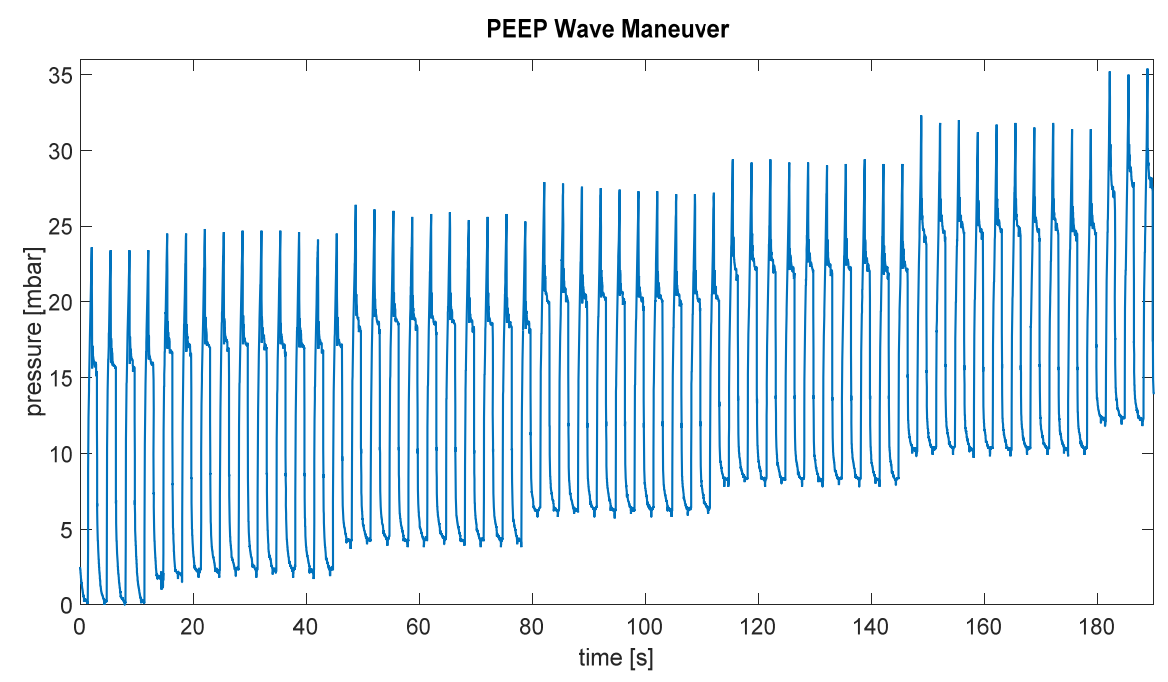

Figure 1. Pressure curve during a PEEP Wave maneuver (patient McREM072).

In physiological modeling the simplest model to describe the behavior of the respiratory system (lung and airways) is a first order model (FOM) [19], where the airway passage is symbolized by a single (constant) resistance term and the tissue 
behavior regarding the resistance to expansion is described by a constant elastance. The equation of this FOM is shown in Equation (1) and an electrical analogy is shown in Figure 2.

$$
P=E V+R \dot{V}+P_{0}
$$

where: $P$ is the airway pressure, $P_{0}$ is the offset pressure, $V$ is the volume, $\dot{V}$ is the flow, $R$ is the respiratory system resistance and $E$ is the respiratory system elastance.

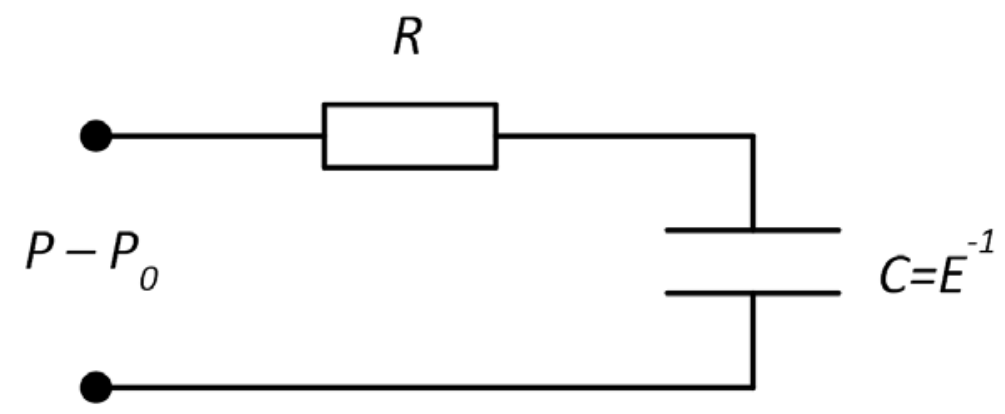

Figure 2. Electrical analogy of the FOM (pulmonary mechanics)

The FOM offers ease of modeling at the cost of the descriptive skill and thus cannot capture the total pressure flow characteristics of the breathing process, but for this study it is sufficient and provides the parameters we are interested in.

\section{Methods}

\subsection{Data}

This study used the McREM clinical data (full details are available at Stahl et al. [12]). In this dataset different respiratory maneuvers were carried out on 28 mechanical ventilated ARDS patients. All patients were ventilated with identical Evita4Lab ventilator systems (Draeger Medical, Lübeck, Germany).

The systems were built up by a standard patient ventilator (Draeger Evita4). The airway gas flow was measured via a non-heated Fleisch No. 2 pneumotachograph (F_G GmbH, Hechingen, Germany), which was calibrated and connected to a differential pressure transducer (PC100 SDSF, Hoffrichter, Schwerin, Germany). To avoid influences of moisture on flow measurement, a heat-moisture exchanger (Aqua_FH, Hudson, Temecula, CA) was placed in between the pneumotachograph and the connector tube. The airway pressure was obtained by a piezoresistive pressure transducer (1790, SI-special instruments, Nördlingen, Germany). 
A control group (McREMO) was included in the study, where pressure, flow and volume data of 10 mechanically ventilated patients under preoperative anesthesia conditions for orthopedic surgery were recorded at the University Hospital in Freiburg. The following paragraph is a brief description of the control group protocol, full details are available at Ganzert et al.[20].

Patients undergoing general anesthesia and tracheal intubation were included in the study. All patients were ventilated in volume-controlled mode in the supine position $(10 \mathrm{~mL} / \mathrm{kg}$, respiratory rate $12 \mathrm{breaths} / \mathrm{min}$, inspiratory: expiratory ratio: 1:1.5, $\mathrm{FiO}_{2}$ : $100 \%$, PEEP $0 \mathrm{cmH}_{2} \mathrm{O}$ ). To avoid atelectasis, a PEEP wave recruitment maneuver was performed with PEEP-steps up, until a plateau pressure of $45 \mathrm{cmH}_{2} \mathrm{O}$ was reached. Each corresponding PEEP level was maintained for six breaths and after the maneuver the PEEP level was reduced to ZEEP. After the incremental PEEP wave maneuver, a super-syringe maneuver was done. After both maneuvers the patients were ventilated on ZEEP-level for at least five minutes.

Both patient studies (ARDS group, control group) were approved by the local ethics committees (details in Stahl et al. [12] and Ganzert et al. [20]) and provide flow, volume and pressure data.

\subsection{Modelling}

To obtain the respiratory system elastance and resistance for each breath, we limited the model fitting to only the inspiration phase of the breathing cycles, excluding the end-inspiratory break. Thus, it was sufficient to use a FOM, which simply delivers the two desired parameters (Equation (2b)). This linear regression problem was solved using the MATLAB backslash function (R2016a, The MathWorks, Natick, USA).

with

$$
\begin{gathered}
\boldsymbol{A} x=b \\
\boldsymbol{A}=\left[\begin{array}{ll}
\vec{V} & \overrightarrow{\dot{V}}
\end{array}\right], \quad \vec{b}=\vec{P}-P_{0} \text { and } x=\left(\begin{array}{c}
E \\
R
\end{array}\right) \\
\left(\begin{array}{l}
E \\
R
\end{array}\right)=\boldsymbol{A} \backslash \vec{b}
\end{gathered}
$$

After the determination of elastance and resistance values (Figure 3), we analyzed the effect of the PEEP wave maneuver. Therefore, the elastance before $\left(E_{1}\right)$ and after the maneuver $\left(E_{2}\right)$ were compared (Figure 4 and Equation (3)). The dynamic behavior was investigated by fitting Equation (4) to the elastance curve.

$$
\begin{gathered}
\Delta E_{p w}=E_{1}-E_{2} \\
E(t)=E_{2}\left(1-b \cdot e^{-\frac{t}{T}}\right)
\end{gathered}
$$

where: $E_{1}$ is the elastance before the maneuver, $E(t)$ the elastance curve after the maneuver, $E_{2}$ the final value of the elastance after the maneuver, $\Delta E_{p w}$ the elastance change due to the PEEP wave maneuver, $b$ a constant factor, $T$ a time constant and $t$ is the time after the maneuver. 


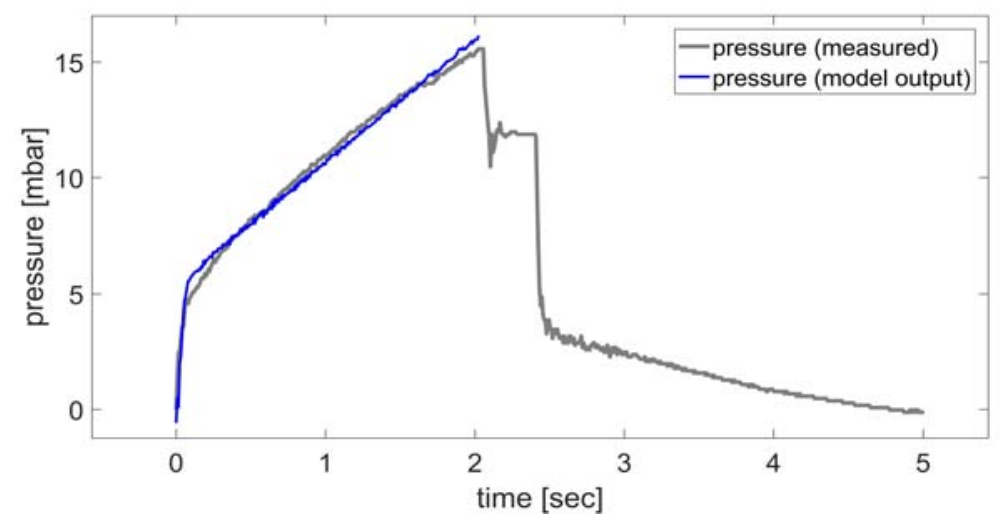

Figure 3. Model output after determination of the resistance and elastance values. The model fit was limited to the inspiratory part of the breath.

These investigations were done for all patients of the McREM (ARDS) and McREMO (control group) dataset. Afterwards an unpaired two-sample ttest was performed (ttest2 function in MATLAB) to see if there is a significant difference between the ARDS and the control group.

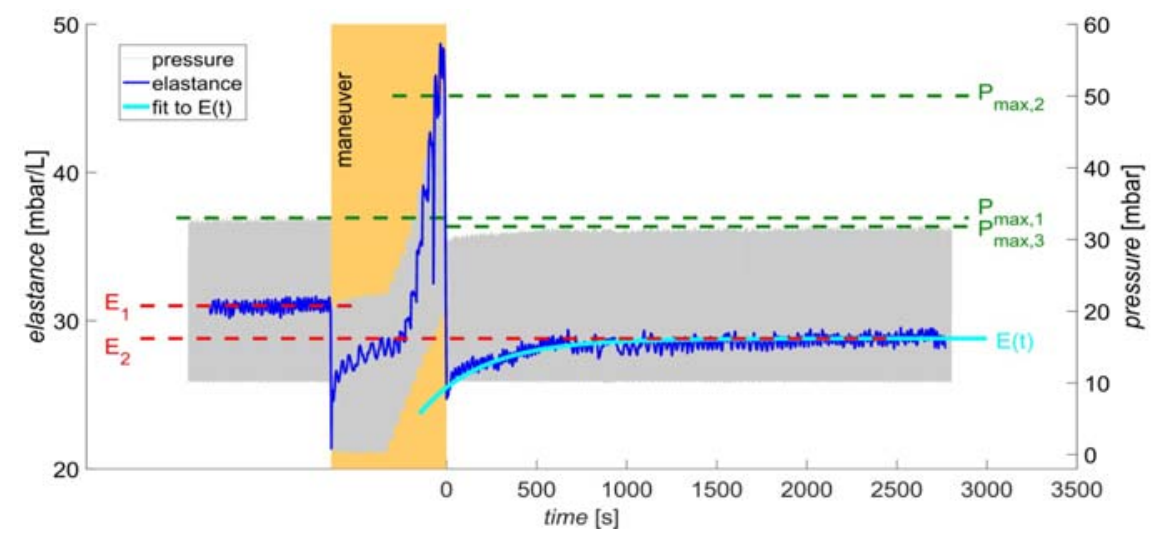

Figure 4. ARDS Group-patient McREM09: Changes in $E(t)$ after a recruitment maneuver (PEEP wave maneuver). The upper red dashed line is the mean value $E_{1}$ of the elastance before the maneuver, the lower red dashed line is final value of the elastance $E_{2}$ sometime after the maneuver.

\section{Results}

In 17 of 28 ARDS patients' data PEEP wave maneuvers lead to a reduction of the elastance of the lung tissue, while in 8 of 28 patients' data an increase of the elastance can be observed. In the remaining three datasets, the parameter identification method delivered implausible elastance values, and the results were thus excluded from this analysis. 
In the control group (lung healthy patients), 9 of 10 patients' data exhibited an increase of the elastance after the PEEP wave maneuver. In 8 of 10 datasets of the control group an initial reduction of the elastance $\Delta E_{d m}$ during the PEEP wave maneuver can be seen (up to $24 \%$ of the elastance $E_{l}$ before the maneuver), followed by an increase of elastance in higher pressure levels (Figure 5).

$$
\Delta E_{d m}=E_{1}-\min (E(t))
$$

This initial decrease cannot be found during PEEP wave maneuvers in ARDS patients' data, where the elastance increases at each PEEP step (Figure 6).

The results of this analysis are listed in Table 1. The median value of $T$ is $\tilde{T}=$ $25 \mathrm{~s}$ (Interquartile range $(\mathrm{IQR})=42.3 \mathrm{~s})$ in the control group while $\tilde{T}=141 \mathrm{~s}$ $(\mathrm{IQR}=168 \mathrm{~s})$ in the ARDS group. A significant $\mathrm{p}$-value of 0.014 points out, that there is a significant difference between these two groups. Unfortunately, the time constant $T$ and therefore the dynamic behavior could be determined and analyzed only in $12 / 28$ patients of the ARDS group and in 8/10 patients of the control group. The other datasets contained insufficient dynamics to properly define the exponential curve of Equation (4).

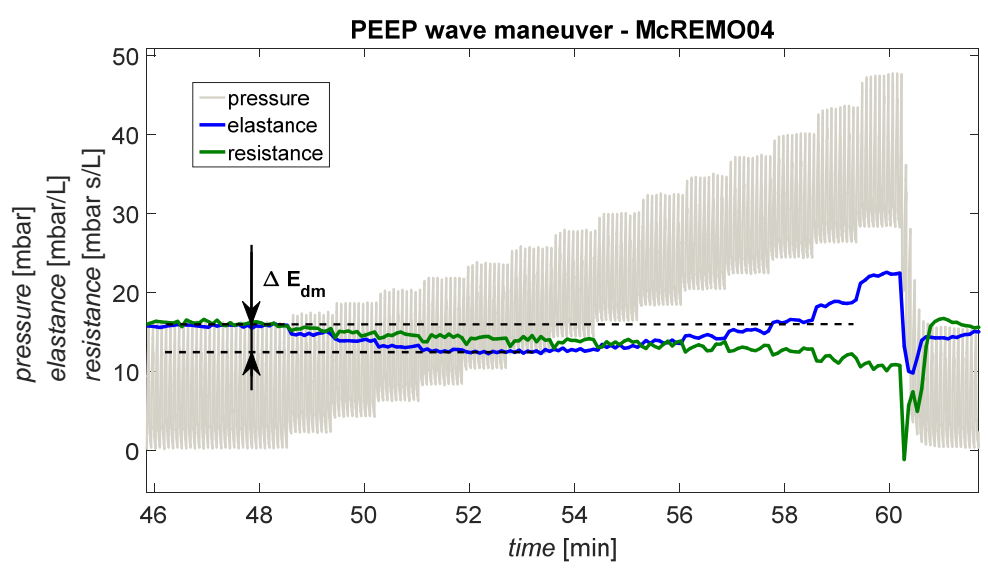

Figure 5. The elastance and resistance trend during a PEEP wave maneuver for lung healthy patients (McREMO04).

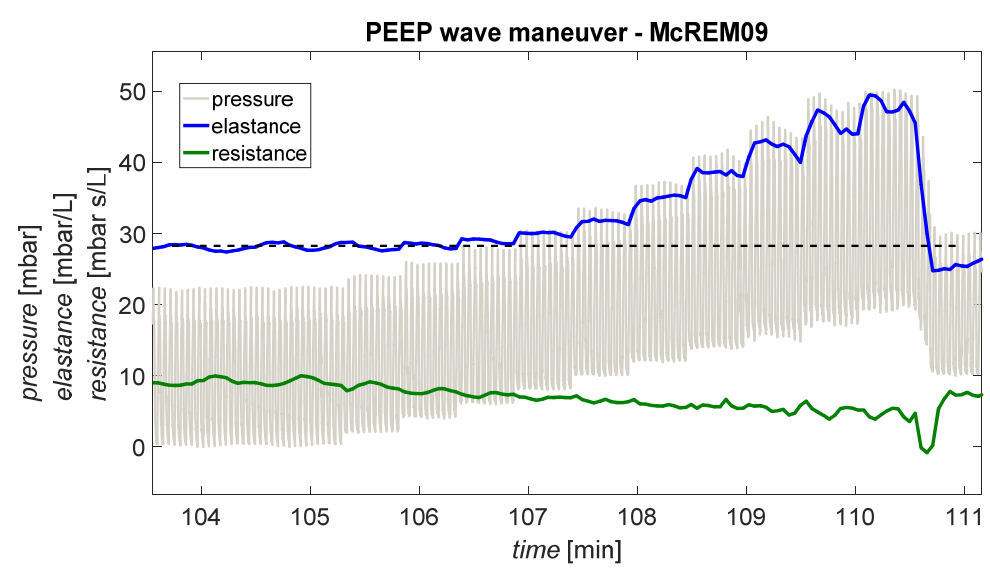

Figure 6. The elastance and resistance trend during a PEEP wave maneuver for ARDS patients (McREM009) 
Table 1. Parameter changes due to PEEP wave maneuver

\begin{tabular}{|c|c|c|c|c|c|}
\hline Patient & $\begin{array}{c}E_{1} \\
{[\mathrm{mbar} / \mathrm{L}]}\end{array}$ & $\begin{array}{c}E_{2} \\
{[\mathrm{mbar} / \mathrm{L}]}\end{array}$ & $\begin{array}{c}\Delta E_{p w} \\
{[\mathrm{mbar} / \mathrm{L}]}\end{array}$ & $\begin{array}{c}T \\
{[\mathrm{~s}]}\end{array}$ & $\begin{array}{c}\Delta E_{d m} \\
{[\mathrm{mbar} / \mathrm{L}]}\end{array}$ \\
\hline \multicolumn{6}{|c|}{ ARDS Group } \\
\hline McREM009 & 30.9 & 28.7 & 2.2 & 370 & - \\
\hline McREM011 & 36.4 & 34.0 & 2.4 & 74 & - \\
\hline McREM012 & 16.8 & 16.9 & -0.1 & 106 & - \\
\hline McREM063 & 35.3 & 37.3 & -2.0 & 242 & - \\
\hline McREM075 & 23.7 & 24.0 & -0.3 & 490 & - \\
\hline McREM078 & 21.8 & 20.0 & 1.8 & 211 & - \\
\hline McREM088 & 34.7 & 34.5 & 0.2 & 145 & - \\
\hline McREM090 & 13.3 & 14.6 & -1.3 & 600 & - \\
\hline McREM093 & 18.5 & 17.7 & 0.8 & 20 & - \\
\hline McREM108 & 33.9 & 31.5 & 2.4 & 80 & - \\
\hline McREM111 & 48.0 & 50.5 & -2.5 & 141 & - \\
\hline McREM117 & 23.3 & 18.4 & 4.9 & 20 & - \\
\hline \multicolumn{6}{|c|}{ Control Group } \\
\hline McREMO04 & 15.6 & 15.0 & 0.6 & 10 & 3.3 \\
\hline McREMO05 & 13.8 & 14.1 & -0.3 & 40 & 3.3 \\
\hline McREMO08 & 12.1 & 12.4 & -0.3 & 14 & 1.0 \\
\hline McREMO09 & 9.1 & 13.2 & -4.1 & 136 & - \\
\hline McREMO10 & 15.0 & 16.0 & -1.0 & 8 & 1.0 \\
\hline McREMO11 & 15.4 & 15.6 & -0.2 & 9 & 2.9 \\
\hline McREMO12 & 5.9 & 6.9 & -1.0 & 36 & 1.8 \\
\hline McREMO14 & 14.1 & 14.5 & -0.4 & 88 & 0.3 \\
\hline
\end{tabular}

\section{Discussion}

The FOM shows that a PEEP wave maneuver can reduce the respiratory system elastance and thus reduce the driving pressure needed for the mechanical ventilation of patients with ARDS to maintain a fixed tidal volume. Most of the patients' data showed a moderate decrease of the elastance as a result of the PEEP wave maneuver, which comes along with a reduction of the airway pressure in volume controlled ventilation (Equation (1)). A lower airway pressure in delivering a constant tidal volume is the goal of the common treatment of patients with ARDS as noted in the lung protective ventilation. The lower airway pressure reduces the risks of further lung damage due to excessive pressure [21] [22].

Recruitment maneuvers can recruit pulmonary regions that are collapsed due to illness. In the McREM dataset, multiple maneuvers were performed in random sequence and thus, the effect of the PEEP wave maneuver cannot be determined quantitatively. When some lung regions are recruited and collapsed alveoli are already reopened by a prior recruitment maneuver, the outcome of a subsequent 
PEEP wave maneuver will be reduced. However, in most patients' data, a positive effect of the PEEP wave maneuver can be seen. Some patients show a reduction of the elastance $E_{2}$ of up to $27 \%$ of the initial elastance $E_{1}$, while others show minor changes.

To compare the dynamic behavior of the elastance $E(t)$ after the maneuver of the ARDS and the control group, we were able to determine $T$ in 12 of the 28 patients' data. In the control group, $T$ could be determined in 8 of 10 patients. The contrast across ARDS patients and healthy volunteers can be seen in Table 1. Overall, the faster response of the control group is obvious. Thus, healthy lung tissue can adapt significantly faster to changes, but this hypothesis should be proven in another dataset, where a defined sequence of the maneuvers could bring deeper understanding.

The reduction of the elastance during the PEEP wave maneuver $\left(\Delta E_{d m}\right)$ in the control group shows that healthy lungs are easily capable in handling a certain increase in airway pressure, which could be due to recruitment of unused or collapsed (due to sedation) lung regions. Then, further increase of airway pressure leads to an increase of the elastance, which can be seen as a sign of a starting over-distention of the alveoli. Unfortunately, this reserve capacity of the lung cannot be seen in the data of ARDS patients, where each increase of pressure yields an increase of the lung elastance. Existing lung damage is initially balanced with recruitment of the reserve capacity up to a point where the damage is advanced and cannot be regulated anymore. Hence damaging over-distention of pulmonary tissue mitigates the benefit of added recruitment at the higher pressure.

In addition, this investigation confirmed that the airway resistance varies with the airway pressure [23]. The resistance is higher in lower pressure ranges, while in higher airway pressures, the resistance is smaller. This can be observed during all PEEP wave maneuvers-for both the ARDS and control group (e.g. see Figure 5 and Figure 6).

\section{Conclusion}

PEEP wave maneuvers in combination with lung protective ventilation are an evaluated treatment of patients with ARDS. This study showed that the reduction of the elastance due to the PEEP wave maneuver can be observed in most ARDS patients. Additionally, the reduction of the elastance during the maneuver in case of lung healthy patients could potentially be used to diagnose beginning lung injuries. The dynamic behavior after the maneuver should be investigated in future studies, where maneuvers are performed in a set and defined sequence.

\section{Acknowledgements}

Partial support by the EU-Project - "eTime" - ID : "FP7-PEOPLE-2012-IRSES" is gratefully acknowledged. 


\section{References}

[1] Ashbaugh, D., Boyd Bigelow, D., Petty, T., and Levine, B. (1967) Acute Respiratory Distress in Adults. The Lancet, 290, 319-323.

https://doi.org/10.1016/S0140-6736(67)90168-7

[2] Ware, L.B. and Matthay, M.A. (2000) TheAcute Respiratory Distress Syndrome. $N$ Engl J Med, 342, 1334-49.https://doi.org/10.1056/NEJM200005043421806

[3] Silversides, J. and Ferguson, N. (2013) Clinical Review: Acute Respiratory Distress Syndrome - Clinical Ventilator Management and Adjunct Therapy. Critical Care, 17, 225.https://doi.org/10.1186/cc11867

[4] Donahoe, M. (2011) Acute Respiratory Distress Syndrome: A Clinical Review. Pulmonary Circulation, 1, 192-211. https://doi.org/10.4103/2045-8932.83454

[5] The Acute Respiratory Distress Syndrome Network. (2000) Ventilation with Lower Tidal Volumes as Compared with Traditional Tidal Volumes for Acute Lung Injury and the Acute Respiratory Distress Syndrome. New England Journal of Medicine, 342, 1301-1308. https://doi.org/10.1056/NEJM200005043421801

[6] Afifi, S. (2002) Lung Protective Ventilation Strategies. Seminars in Cardiothoracic and Vascular Anesthesia, 6, 259-269. https://doi.org/10.1177/108925320200600308

[7] Halter, J. M., Steinberg, J.M., Schiller, H.J., DaSilva, M., Gatto, L.A., Landas, S., et al.(2003) Positive End-Expiratory Pressure after a Recruitment Maneuver Prevents Both Alveolar Collapse and Recruitment/Derecruitment. Am J RespirCrit Care Med, 167, 1620-1626.https://doi.org/10.1164/rccm.200205-435OC

[8] Lellouche, F. and Lipes, J. (2013) Prophylactic Protective Ventilation: Lower Tidal Volumes for All Critically Ill Patients? Intensive Care Medicine, 39, 6-15. https://doi.org/10.1007/s00134-012-2728-4

[9] Brower, R.G., Lanken, P.N., MacIntyre, N., Matthay, M.A., Morris, A., Ancukiewicz, M., et al. (2004) Higher versus Lower Positive End-Expiratory Pressures in Patients with the Acute Respiratory Distress Syndrome. $N$ Engl $J$ Med, 351, 327-336.https://doi.org/10.1056/NEJMoa032193

[10] Kacmarek, R.M. and Villar, J. (2011) Lung recruitment maneuvers during acute respiratory distress syndrome: is it useful? Minerva Anestesiologica, 77, 85-89.

[11] Villagra, A., Ochagavia, A., Vatua, S., Murias, G., Del Mar Fernandez, M., Lopez Aguilar, J., et al. (2002) Recruitment Maneuvers during Lung Protective Ventilation in Acute Respiratory Distress Syndrome. Am J RespirCrit Care Med, 165, 165-70. https://doi.org/10.1164/ajrccm.165.2.2104092

[12] Stahl, C.A., Moller, K., Schumann, S., Kuhlen, R., Sydow, M., Putensen, C., et al.(2006) Dynamic Versus Static Respiratory Mechanics in Acute Lung Injury and Acute Respiratory Distress Syndrome. Crit Care Med, 34, 2090-2098. https://doi.org/10.1097/01.CCM.0000227220.67613.0D

[13] Putensen, C., Baum, M., Koller, W., and Putz, G. (1989) The PEEP Wave: An Automated Technic for Bedside Determination of the Volume/Pressure Ratio in the Lungs of Ventilated Patients. Anaesthesist, 38, 214-9.

[14] Schranz, C. (2013) Individualisierung Von Modellsystemen Zur Unterstützung Von Therapiemaßnahmen, Klinik für Anästhesiologie und Intensivpflege, Dresden University of Technology, Dresden.

[15] Amato, M.B.P., Barbas, C.S.V., Medeiros, D.M., Magaldi, R.B., Schettino, G.P., Lorenzi-Filho, G., et al.(1998) Effect of a Protective-Ventilation Strategy on Mortality in the Acute Respiratory Distress Syndrome. N Engl J Med, 338, 347-354. https://doi.org/10.1056/NEJM199802053380602

[16] Rees, S., Allerød, C., Murley, D., Zhao, Y., Smith, B., Kjærgaard, S., et al. (2006) Using Physiological Models and Decision Theory for Selecting Appropriate Ventilator Settings. Journal of Clinical Monitoring and Computing, 20, 421-429. https://doi.org/10.1007/s10877-006-9049-5

[17] Kretschmer, J., Becher, T., Riedlinger, A., Schadler, D., Weiler, N., and Moller, K. (2013) A Simple Gas Exchange Model Predicting Arterial Oxygen Content for Various FiO2 Levels. Conference Proceedings IEEE Eng Med BiolSoc, 465-468. https://doi.org/10.1109/EMBC.2013.6609537

[18] Schranz, C., Becher, T., Schadler, D., Weiler, N., and Möller, K. (2014) Model-based Setting of Inspiratory Pressure and Respiratory Rate in Pressure-ControlledVentilation. PhysiolMeas, 35, 383-397.

https://doi.org/10.1088/0967-3334/35/3/383

[19] Cobelli, C. (2008) Introduction to Modeling in Physiology and Medicine. Academic Press, Amsterdam; Boston. 
[20] Ganzert, S., Möller, K., Steinmann, D., Schumann, S., and Guttmann, J. (2009) Pressure-dependent Stress Relaxation in Acute Respiratory Distress Syndrome and Healthy Lungs: An Investigation Based on a Viscoelastic Model. Crit Care, 13.https://doi.org/10.1186/cc8203

[21] Meade, M.O., Cook, D.J., Griffith, L.E., Hand, L.E., Lapinsky, S.E., Stewart, T.E., et al. (2008) A Study of the Physiologic Responses to a Lung Recruitment Maneuver in Acute Lung Injury and Acute Respiratory Distress Syndrome. Respiratory Care, 53, 1441-1449.

[22] Stewart, T.E., Meade, M.O., Cook, D.J., Granton, J.T., Hodder, R.V., Lapinsky, S.E., et al. (1998) Evaluation of a Ventilation Strategy to Prevent Barotrauma in Patients at High Risk for Acute Respiratory Distress Syndrome. N Engl J Med, 338, 355-361.https://doi.org/10.1056/NEJM199802053380603

[23] Laufer, B., Kretschmer, J., Docherty, P.D., Chiew, Y.S., and Möller, K. (2016) The Influence of Airway Resistance in the Dynamic Elastance Model. In: E. Kyriacou, et al., Eds., XIV Mediterranean Conference on Medical and Biological Engineering and Computing 2016, Springer International Publishing, Cham, 56-61. https://doi.org/10.1007/978-3-319-32703-7 12

\section{Submit or recommend next manuscript to SCIRP and we will provide best service for you:}

Accepting pre-submission inquiries through Email, Facebook, LinkedIn, Twitter, etc. A wide selection of journals (inclusive of 9 subjects, more than 200 journals)

Providing 24-hour high-quality service

User-friendly online submission system

Fair and swift peer-review system

Efficient typesetting and proofreading procedure

Display of the result of downloads and visits, as well as the number of cited articles

Maximum dissemination of your research work

Submit your manuscript at: http://papersubmission.scirp.org/

Or contact jbise@scirp.org 